\title{
Modulation of the Schottky Barrier Height for CMOS advanced contacts
}

\author{
Mariela A. Menghini ${ }^{\mathrm{a}, *}$, Pia Homm ${ }^{\mathrm{a}}$, Chen-Yi Su ${ }^{\mathrm{a}}$, Jorge A. Kittl ${ }^{\mathrm{b}}$, Ryuji Tomita ${ }^{\mathrm{c}}$, Ganesh Hegde ${ }^{\mathrm{b}}$, \\ Joon-Gon Lee ${ }^{c}$, Sangjin Hyun ${ }^{c}$, Chris Bowen ${ }^{b}$, Mark.S. Rodder ${ }^{b}$, Valeri Afanas'ev ${ }^{a}$, Jean-Pierre Locquet ${ }^{a}$ \\ a Dept. of Physics and Astronomy, KU Leuven, Leuven, Belgium \\ b Advanced Logic Lab, Samsung Semiconductor Inc., Austin, TX, USA \\ c Semiconductor Research and Development, Samsung, Dongtan, Republic of Korea
}

\section{A R T I C L E I N F O}

\section{Article history:}

Received 15 June 2015

Received in revised form 7 January 2016

Accepted 8 January 2016

Available online $\mathrm{xxxx}$

\section{Keywords:}

Schottky Barrier

Mosfet

SiGe and Ge channel

Contact resistance

Capacitance measurements

\begin{abstract}
A B S T R A C T
Contact schemes for scaled Si, SiGe and Ge channel MOSFET devices are discussed, consistent with an approach based on SiGe alloys with low Schottky Barrier Height (SBH) for pMOS and Si contacts for nMOS, making reduction of the SBH to nSi critical. Contacts to $\mathrm{n}+\mathrm{Ge}$ with contact resistivity $\left(\rho_{\mathrm{c}}\right)$ values of $\sim 1.5 \times 10^{-8} \Omega \mathrm{cm}^{2}$ are achieved using $n+$ Si passivation. Further, methods for SBH reduction to $\mathrm{nSi}$, and their underlying mechanisms, are studied. Accurate cryogenic CV measurements were used to extract SBH. We show that chalcogenide segregation can be effective in lowering the SBH by a dipole effect, while MIS contacts have a partial un-pinning effect leading to $\mathrm{SBH}=0.00 \pm 0.01 \mathrm{eV}$.
\end{abstract}

(c) 2016 Elsevier B.V. All rights reserved.

\section{Introduction}

As MOS device dimensions are scaled towards the $7 \mathrm{~nm}$ node and beyond, the impact of parasitics such as contact resistance on device performance is becoming dominant. Specifically, the metal-semiconductor interface resistance is becoming a large portion of the total device on resistance. Thus, methods to achieve very low metal-semiconductor interface specific contact resistivities $\left(\rho_{\mathrm{c}}\right)$ are needed for future nodes. We consider contact schemes for devices with SiGe channels (considering all the range from pure Si to pure $\mathrm{Ge}$ ). Strong Fermi Level Pinning (FLP) close to the Valence Band Edge (VBE) resulting in low Schottky Barrier Height $(\mathrm{SBH})$ contacts to $\mathrm{p}+\mathrm{Ge}$, has been reported [1,2]. Studies of the effect of Ge content on FLP indicate that strong FLP is already present in SiGe at Ge contents $>20 \%$ [2]. Thus, very low resistivity contacts to $\mathrm{p}+\mathrm{SiGe}\left(30 \% \mathrm{Ge}, \rho_{\mathrm{c}}=1.5 \times 10^{-9} \Omega \mathrm{cm}^{2}\right)$ [3] and $\mathrm{p}+\mathrm{Ge}$ $\left(\rho_{\mathrm{c}}=2.3 \times 10^{-9} \Omega \mathrm{cm}^{2}\right)$ [4] have been reported, appropriate for contact solutions for SiGe channel pMOS devices. For nMOS devices, improvements are still needed. Contacts for Ge channel nMOS devices are problematic due to unfavorable FLP and difficulty to obtain high activation in nGe. Pulsed Laser Activation (LA) has been known to allow very high metastable dopant activation beyond equilibrium solubility, but requires the melting of layers [5]. Recently, low $\rho_{\mathrm{c}}\left(1.9 \times 10^{-8} \Omega \mathrm{cm}^{2}\right)$ to $\mathrm{n}+\mathrm{Ge}$ was achieved using pulsed LA [4]. However, integration of pulsed LA processes has been problematic, and more so for complex geometries

\footnotetext{
* Corresponding author.

E-mail address: mariela.menghini@fys.kuleuven.be (M.A. Menghini).
}

as found in fin or nanowire devices expected for future nodes. In this paper, we present studies for contact approaches which can be integrated readily, for SiGe nMOS channel devices, using an $n+$ Si contacted surface. A key parameter for further improving contacts using this approach is the reduction of $\mathrm{SBH}$ to $\mathrm{n}+\mathrm{Si}$. Thus studies of $\mathrm{SBH}$ modulation and its origin are presented in this work.

\section{Experiments}

SBHs were obtained from Cryogenic CV measurements (at $77 \mathrm{~K}$ ) performed using a Lakeshore CPX-VF cryogenic probe station. CV SBH characterization offers advantages over IV characterization in that Richardson constants are not needed for the extraction, and are less prone to artifacts and effects that deter accurate SBH determination in IV characterization such as the effect of interface defects [6]. The advantage of low temperature CV measurements is that it reduces the leakage currents allowing for accurate measurements to be extended to the voltages close to the flatband condition. We also note that the CV measurements are not affected by dynamic effects such as image force barrier lowering present in IV measurements, thus allowing an extraction of the intrinsic SBH, $\Phi_{B}$. The Schottky Barrier Height for n-type semiconductor $(\mathrm{nSBH})$ is obtained by:

$\Phi_{B}=\mathrm{V}_{\mathrm{i}}+\frac{\mathrm{kT}}{\mathrm{q}} \ln \left(\frac{\mathrm{N}_{\mathrm{C}}(\mathrm{T})}{\mathrm{N}_{\mathrm{D}}}\right)+\frac{\mathrm{kT}}{\mathrm{q}}$ 


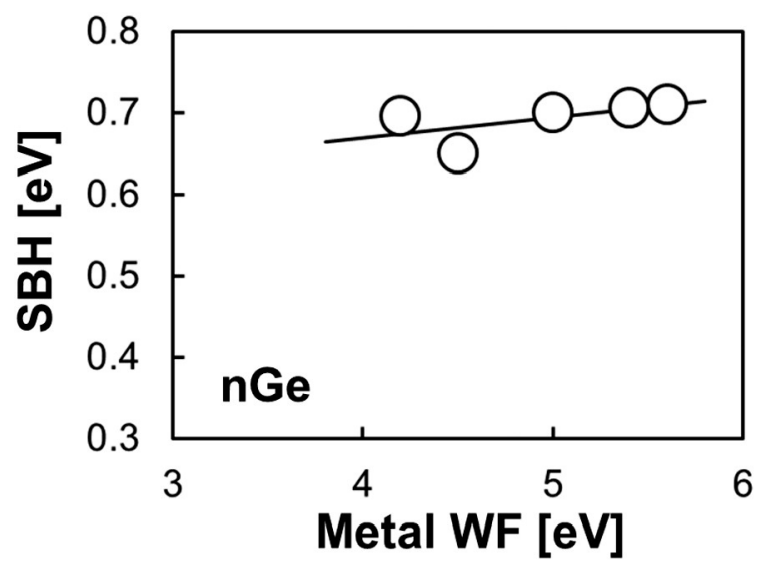

Fig. 1. Strong FLP on nGe results in high SBH independently of metal WF.

where $\mathrm{V}_{\mathrm{i}}$ and $\mathrm{N}_{\mathrm{D}}$ are calculated from the linear fit of $(\mathrm{A} / \mathrm{C})^{2}$ vs $\mathrm{V}$ :

$\left(\frac{\mathrm{A}}{\mathrm{C}}\right)^{2}=\frac{2\left(\mathrm{~V}_{\mathrm{bi}}-\mathrm{V}-\mathrm{kT} / \mathrm{q}\right)}{\mathrm{q} \mathrm{K}_{\mathrm{S}} \varepsilon_{0} \mathrm{~N}_{\mathrm{D}}}$

where the slope is $a=\frac{-2}{q \mathrm{~K}_{S} \varepsilon_{0} \mathrm{~N}_{\mathrm{D}}}$ and the intercept with the $\mathrm{V}$ axis is $\mathrm{V}_{\mathrm{i}}=$ $\left(\mathrm{V}_{\mathrm{bi}}-\frac{\mathrm{kT}}{\mathrm{q}}\right) . \mathrm{A}, \mathrm{V}, \mathrm{N}_{\mathrm{D}}, \mathrm{V}_{\mathrm{bi}}$, and $\mathrm{K}_{\mathrm{S}} \varepsilon_{0}$ are the contact area, the applied voltage, the donor concentration, the built in potential, and the permittivity of the semiconductor, respectively. Errors in SBH extracted, for data that shows good linearity, are as low as $10 \mathrm{meV}$. Specific contact resistivities were extracted using nano-transmission line measurement (TLM) structures with contact sizes of $\sim 50 \mathrm{~nm}$ and separation between contacts from $\sim 20 \mathrm{~nm}$. The reported $\rho_{\mathrm{c}}$ values are, as in typical TLM analysis, extracted from extrapolations to 0 contact separation, and without corrections from additional parasitics due to metal and/or additional semiconductor layers at each end (thus overestimating the actual interface $\rho_{\mathrm{c}}$ values by $\left.2-4 \times 10^{-9} \Omega \mathrm{cm}^{2}\right)$.

\section{Results and discussion}

\subsection{Contact scheme for Si, SiGe, Ge channel nMOS devices}

With strong FLP close to the VBE, SBH of metal contacts to nGe is very high (Fig. 1), regardless of the metal workfunction (WF), making it difficult to achieve low resistivity contacts. Due to the good alignment of the conduction band edge (CBE) of Si and Ge, solutions using contacts to $\mathrm{n}+\mathrm{Si}$ surfaces to access SiGe or Ge channels in nMOS devices have been proposed [7,8]. Initial studies achieved $\rho_{\mathrm{c}} \sim 1 \times 10^{-6} \Omega \mathrm{cm}^{2}$,

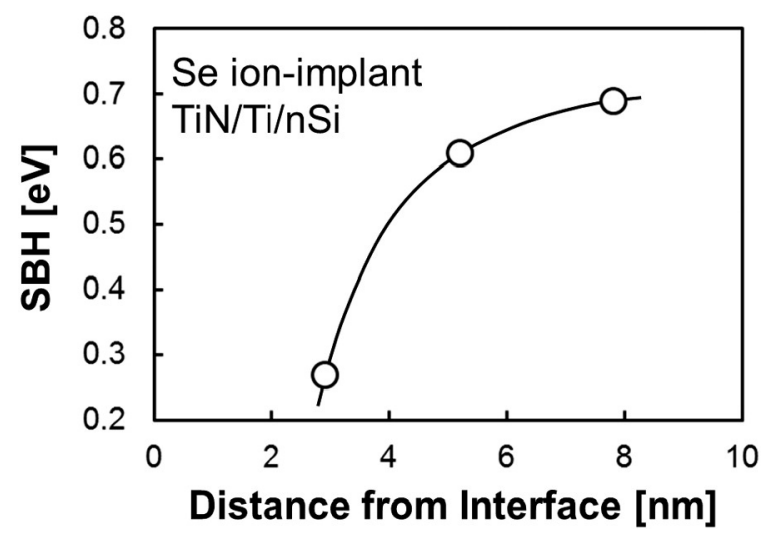

Fig. 3. SBH vs position of II for chalcogenide segregation.

which was limited by dopant activation levels $\left(\sim 1 \times 10^{20} \mathrm{~cm}^{-3}\right)$ and a non-optimized contact process to the $\mathrm{n}+$ Si surface $[7,8]$.

A similar scheme of $\mathrm{n}+$ Si passivation of $\mathrm{n}+\mathrm{Ge}$, with improvements on dopant activation of the ISD epi $\mathrm{n}+$ Si layer $\left(>3 \times 10^{20} \mathrm{~cm}^{-3}\right)$ and the metal contact process led to $\rho_{\mathrm{c}}$ values of $\sim 1.5 \times 10^{-8} \Omega \mathrm{cm}^{2}$ (Fig. 2), without the use of a melt laser process. Note that the nano-TLM structures used in this study force the current from the $(\sim 20 \mathrm{~nm}) \mathrm{n}+\mathrm{Si}$ epi on the contact regions to the connecting underlying $(\sim 50 \mathrm{~nm}) \mathrm{n}+$ Ge layer.

These results indicate that further reduction of contact resistivity to $\mathrm{n}+$ Si can be beneficial and applied, not only to Si-channel but also to SiGe and Ge channel nMOS devices. A restriction is that the activation methods need to be compatible with the thermal budget limitations of the SiGe alloy or Ge present in the channel. A parameter that can be very effective in lowering the contact resistivity to $\mathrm{n}+\mathrm{Si}$ and can relieve the requirements on ultra-high dopant activation is the SBH. Thus, methods to reduce the $\mathrm{SBH}$ to $\mathrm{n}+\mathrm{Si}$ are studied in the next sections.

\subsection{Chalcogenide segregation}

SBH lowering by chalcogenide segregation on nSi and nGe have been previously studied, with SBH determined by IV techniques [13-15]. Here, we studied the effects of Se ion implants (II) on SBH to nSi by low temperature CV measurements, and analyzed the nature of the SBH lowering. Fig. 3 shows the sensitivity of the SBH lowering to the distance of the II projected range from the metal-Si interface. Lower SBHs are achieved when the peak position of the II is closer to the interface. Fig. 4 shows the effect of total implanted dose on SBH. It is clear that much less than a monolayer (ML) of Se is sufficient for large SBH reductions, particularly when the Se is implanted close to the interface. In Fig. 5, we show the effect of chalcogenide segregation from experiments

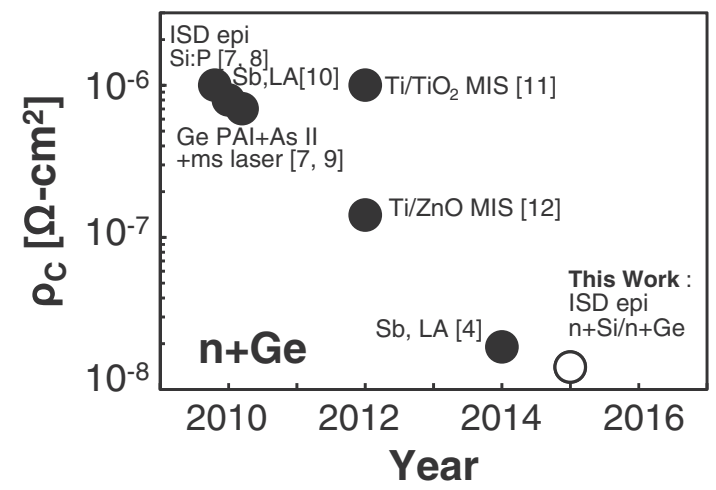

Fig. 2. Comparison of contact resistivity obtained in the present work with the values reported in the literature ([4,7-12]) for different contact schemes to $\mathrm{n}+\mathrm{Ge}$.

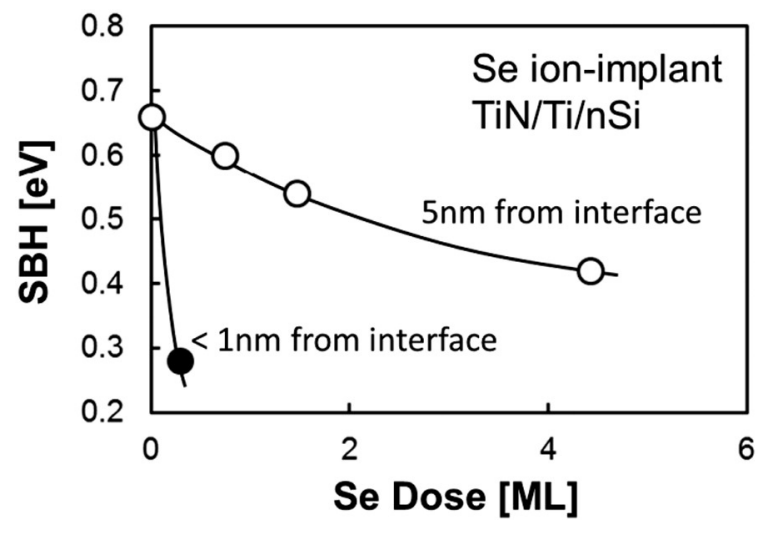

Fig. 4. SBH vs II dose for chalcogenide segregation. 


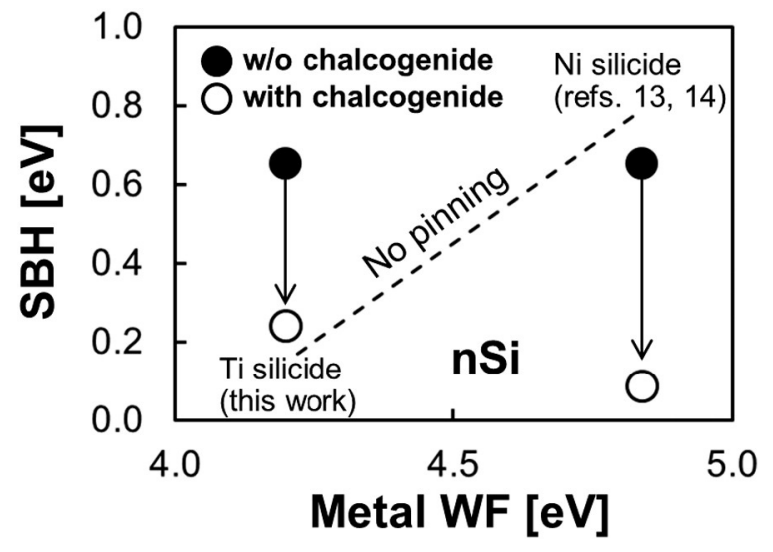

Fig. 5. Impact of chalcogenide segregation on SBH for metals of different WF.

in this work and from literature [13,14]. All experiments, both for $\mathrm{S}$ and Se performed on $\mathrm{Si}, \mathrm{Ge}$ and $\mathrm{SiC}$ substrates have consistently shown a decrease in SBH towards very low values. Fig. 5 is clearly inconsistent with an effect of the chalcogenide un-pinning the Fermi Level. FL un-pinning would result in data points moving towards the dotted line in Fig. 5 (labeled “No pinning"). This is clearly inconsistent with experimental data. Thus, FL un-pinning is ruled out as possible cause of the SBH modulation with chalcogenides. Another possible mechanism is the formation of an MIS type-structure. Given the low chalcogenide doses sufficient to achieve substantial SBH lowering (significantly below 1 monolayer, Fig. 4) this possibility is also ruled out. Dynamic effects such as image force barrier lowering due to increased active dopants can also be disregarded since these are not present in CV measured SBs. This suggests that formation of an interfacial dipole, which is consistent with all the experimental data, is the main cause of the SBH lowering observed with chalcogenides. We further verified the effectiveness of this technique for $\rho_{\mathrm{c}}$ reduction (Fig. 6). Furthermore, initial results from ab-initio transport simulations show a substantial reduction in $\rho_{\mathrm{c}}$, as seen in the experimental data (Fig. 6).

\subsection{Metal-insulator-semiconductor (MIS) contacts}

In recent years, much attention has been given to approaches incorporating a thin insulating film in contacts, between the metal and semiconductor layers; i.e. the metal-insulator-semiconductor (MIS) scheme $[11,12,16,17]$. In this approach, the separation between metal and semiconductor can effectively decouple the metal states from inducing gap states in the semiconductor, effectively reducing or eliminating FLP above a certain insulator thickness (typically $\sim 1 \mathrm{~nm}$ ). The trade-off is typically the addition of an insulating barrier. In order to avoid this additional insulating barrier penalty, insulators with good CBE alignment to the semiconductor are favored, such as $\mathrm{ZnO}$ or $\mathrm{TiO}_{2}$ for $\mathrm{Si}$, SiGe alloys or $\mathrm{Ge}[11,12,16]$. The problem is that FLP can also be present at metal-

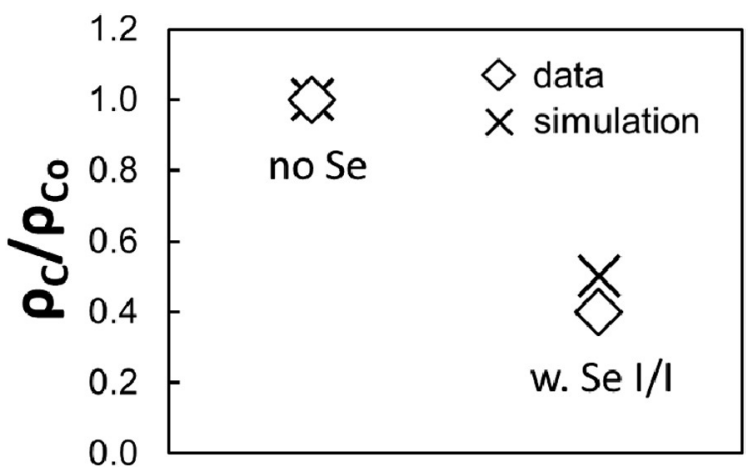

Fig. 6. Effect of chalcogenide segregation on specific interface contact resistivity.

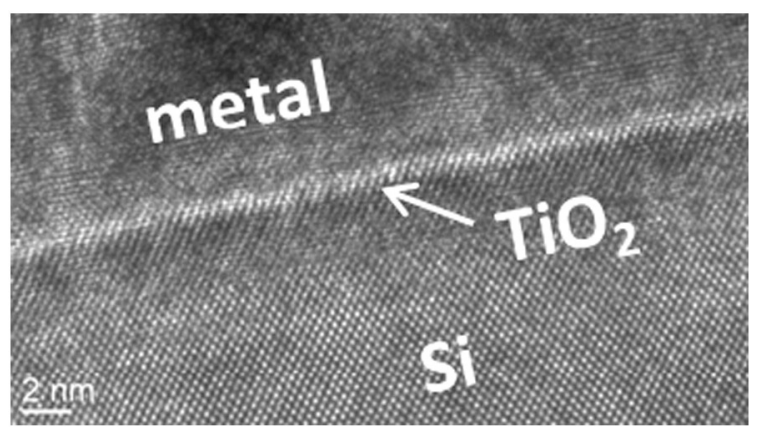

Fig. 7. $\mathrm{TiO}_{2}$ MIS contact structure.

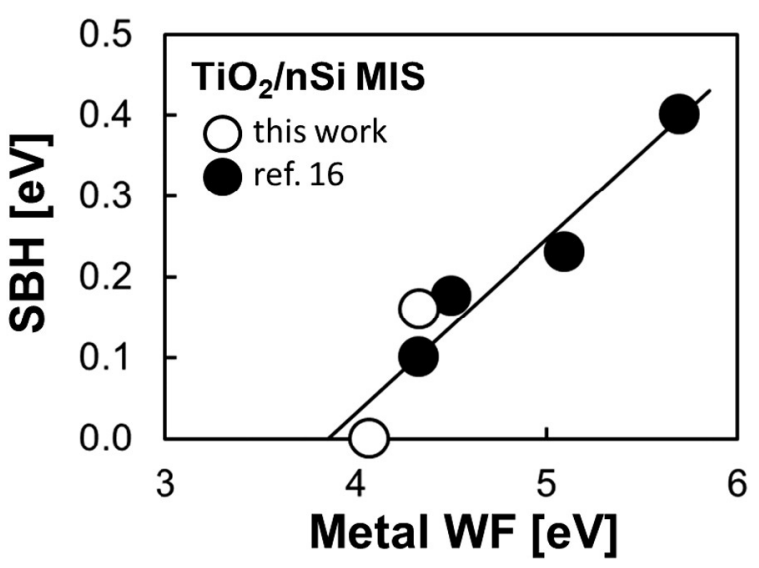

Fig. 8. $\mathrm{SBH}$ vs metal WF for $\mathrm{TiO}_{2}$ MIS contacts to nSi.

insulator interfaces, albeit, with a different pinning factor. This is the case for $\mathrm{TiO}_{2}$ MIS contacts to $\mathrm{nSi}$ [16]. The introduction of $\mathrm{TiO}_{2}$ changes the pinning factor from $S \sim 0.075$ (nSi) to $S \sim 0.24$ (attributed to FLP of $\mathrm{TiO}_{2}$ ), i.e. the MIS has a partial de-pinning effect. Barriers as low as $0.1 \mathrm{eV}$ were reported for $\mathrm{Ti} / \mathrm{TiO}_{2} / \mathrm{nSi}$ contacts [16]. In this work we further explored the potential of $\mathrm{TiO}_{2}$ MIS contacts to nSi (Fig. 7), taking full advantage of the partial un-pinning by extending it to the case of a low WF metal used typically as an nMOS gate electrode WF layer (Fig. 8). In this case, a truly $0 \mathrm{eV} \mathrm{SBH}$ is achieved (within experimental error of $\sim 10 \mathrm{meV}$ ). The fitting of the cryogenic CV measurements used to determine the SBH shows excellent linearity (which allows such accurate extraction), see Fig. 9.

\section{Conclusions}

In conclusion, we demonstrated contacts to $\mathrm{n}+\mathrm{Ge}$ with $\rho_{\mathrm{c}}$ values of $\sim 1.5 \times 10^{-8} \Omega \mathrm{cm}^{2}$ using $\mathrm{n}+$ Si passivation. We studied methods to

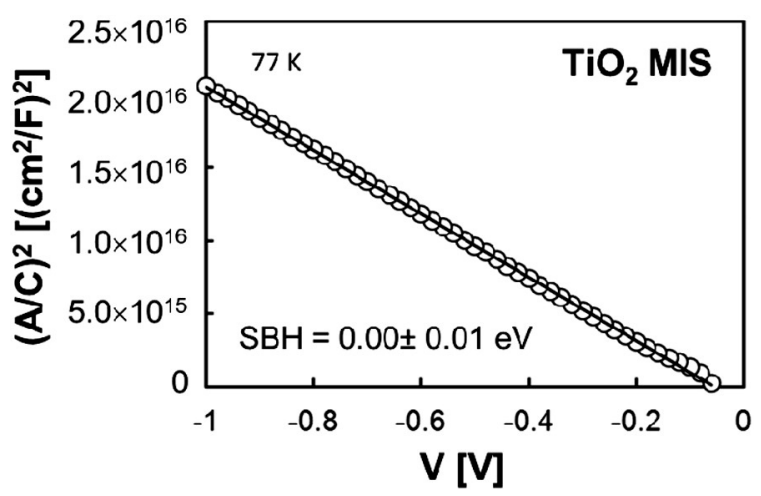

Fig. 9. Extraction of $\mathrm{SBH}$ for $\mathrm{TiO}_{2}$ MIS contacts to nSi using low WF metal, showing $0 \mathrm{SBH}$ achieved. 
further lower the SBH of contacts to $\mathrm{nSi}$, and show that chalcogenide segregation can be effective in lowering the SBH of contacts to nSi by a dipole effect, while MIS contacts have a partial un-pinning effect. $\mathrm{SBH}=0.00 \pm 0.01 \mathrm{eV}$ was achieved. The resultant low SBH contacts to $\mathrm{n}+\mathrm{Si}$ can be used for low resistivity contacts to $\mathrm{Si}$, SiGe and Ge channel devices.

\section{References}

[1] T. Nishimura, K. Kita, A. Toriumi, Appl. Phys. Lett. 91 (2007) 123123.

[2] V. Aubry-Fortuna, et al., J. Appl. Phys. 89 (2001) 5533.

[3] Z. Zhang, et al., Electron Dev. Lett. 34 (2013) 723.

[4] H. Miyoshi, et al., Symp. VLSI Tech. 2014.
[5] J.A. Kittl, et al., Acta Mater. 48 (2000) 4797.

[6] R.R. Lieten, et al., J. Electrochem. Soc. 158 (2011) H358.

[7] K. Martens, et al., IEDM Tech Digest, 2010428.

[8] K. Martens, et al., Appl. Phys. Lett. 98 (2011) 013504.

[9] A. Firrincielli, et al., Appl. Phys. Lett. 99 (2011) 242104.

[10] G. Thareja, et al., IEDM Tech. Digest, 2010245.

[11] J.Y. Jason Lin, et al., Electron Dev. Lett. 33 (2012) 1541.

[12] P.P. Manik, et al., Appl. Phys. Lett. 101 (2012) 182105.

[13] Q.T. Zhao, et al., Appl. Phys. Lett. 86 (2005) 062108.

[14] H.S. Wong, et al., Electron Dev. Lett. 29 (2008) 841.

[15] K. Ikeda, et al., Appl. Phys. Lett. 88 (2006) 152115.

[16] A. Agrawal, et al., Symp. VLSI Tech. 2013.

[17] C.-Y. Su, et al., Jpn. J. Appl. Phys. 53 (2014) 121301. 